\title{
INTEGRAL MEAN ESTIMATES FOR POLYNOMIALS WHOSE ZEROS ARE WITHIN A CIRCLE
}

\author{
K. K. DEWAN, ABDULLAH MIR, and R. S. YADAV
}

(Received 3 November 2000)

\begin{abstract}
Let $p(z)$ be a polynomial of degree $n$ having all its zeros in $|z| \leq k ; k \leq 1$, then for each $r>0, p>1, q>1$ with $p^{-1}+q^{-1}=1$, Aziz and Ahemad (1996) recently proved that $n\left\{\int_{0}^{2 \pi}\left|p\left(e^{i \theta}\right)\right|^{r} d \theta\right\}^{1 / r} \leq\left\{\int_{0}^{2 \pi}\left|1+k e^{i \theta}\right|^{p r} d \theta\right\}^{1 / p r}\left\{\int_{0}^{2 \pi}\left|p^{\prime}\left(e^{i \theta}\right)\right|^{a r} d \theta\right\}^{1 / q r}$. In this paper, we extend the above inequality to the class of polynomials $p(z)=a_{n} z^{n}+$ $\sum_{v=\mu}^{n} a_{n-v} z^{n-v} ; 1 \leq \mu \leq n$ having all its zeros in $|z| \leq k ; k \leq 1$ and obtain a generalization as well as a refinement of the above result.
\end{abstract}

2000 Mathematics Subject Classification. 30A10, 30C10, 30C15.

1. Introduction and statement of results. Let $p(z)$ be a polynomial of degree $n$ and $p^{\prime}(z)$ its derivative. If $p(z)$ has all its zeros in $|z| \leq 1$, then it was shown by Turan [7] that

$$
\max _{|z|=1}\left|p^{\prime}(z)\right| \geq \frac{n}{2} \max _{|z|=1}|p(z)| .
$$

Inequality (1.1) is best possible with equality for $p(z)=\alpha z^{n}+\beta$, where $|\alpha|=|\beta|$. As an extension of (1.1) Malik [4] proved that if $p(z)$ has all its zeros in $|z| \leq k$, where $k \leq 1$, then

$$
\max _{|z|=1}\left|p^{\prime}(z)\right| \geq \frac{n}{1+k} \max _{|z|=1}|p(z)|
$$

Malik [5] obtained a generalization of (1.1) in the sense that the right-hand side of (1.1) is replaced by a factor involving the integral mean of $|p(z)|$ on $|z|=1$. In fact he proved the following theorem.

THEOREM 1.1. If $p(z)$ has all its zeros in $|z| \leq 1$, then for each $r>0$

$$
n\left\{\int_{0}^{2 \pi}\left|p\left(e^{i \theta}\right)\right|^{r} d \theta\right\}^{1 / r} \leq\left\{\int_{0}^{2 \pi}\left|1+e^{i \theta}\right|^{r} d \theta\right\}^{1 / r} \max _{|z|=1}\left|p^{\prime}(z)\right| .
$$

The result is sharp and equality in (1.3) holds for $p(z)=(z+1)^{n}$.

If we let $r \rightarrow \infty$ in (1.3) we get (1.1). Aziz and Ahemad [1] generalized (1.3) in the sense that $\max _{|z|=1}\left|p^{\prime}(z)\right|$ on $|z|=1$ on the right-hand side of (1.3) is replaced by a factor involving the integral mean of $\left|p^{\prime}(z)\right|$ on $|z|=1$ and proved the following result. 
THEOREM 1.2. If $p(z)$ is a polynomial of degree $n$ having all its zeros in $|z| \leq k \leq 1$, then for $r>0, p>1, q>1$ with $1 / p+1 / q=1$,

$$
n\left\{\int_{0}^{2 \pi}\left|p\left(e^{i \theta}\right)\right|^{r} d \theta\right\}^{1 / r} \leq\left\{\int_{0}^{2 \pi}\left|1+k e^{i \theta}\right|^{q r} d \theta\right\}^{1 / q r}\left\{\int_{0}^{2 \pi}\left|p^{\prime}\left(e^{i \theta}\right)\right|^{p r} d \theta\right\}^{1 / p r}
$$

If we let $r \rightarrow \infty$ and $p \rightarrow \infty$ (so that $q \rightarrow 1$ ) in (1.4) we get (1.2).

In this paper, we will first extend Theorem 1.2 to the class of polynomials $p(z)=$ $a_{n} z^{n}+\sum_{v=\mu}^{n} a_{n-v} z^{n-v}, 1 \leq \mu \leq n$, having all the zeros in $|z| \leq k ; k \leq 1$, and thereby obtain a generalization of it. More precisely, we prove the following result.

THEOREM 1.3. If $p(z)=a_{n} z^{n}+\sum_{v=\mu}^{n} a_{n-v} z^{n-v}, 1 \leq \mu \leq n$ is a polynomial of degree $n$ having all its zeros in $|z| \leq k ; k \leq 1$, then for each $r>0, p>1, q>1$ with $1 / p+1 / q=1$,

$$
n\left\{\int_{0}^{2 \pi}\left|p\left(e^{i \theta}\right)\right|^{r} d \theta\right\}^{1 / r} \leq\left\{\int_{0}^{2 \pi}\left|1+k^{\mu} e^{i \theta}\right|^{p r} d \theta\right\}^{1 / p r}\left\{\int_{0}^{2 \pi}\left|p^{\prime}\left(e^{i \theta}\right)\right|^{q r} d \theta\right\}^{1 / q r} .
$$

REMARK 1.4. If we let $r \rightarrow \infty$ and $q \rightarrow \infty$ (so that $p \rightarrow 1$ ) in (1.5) we get (1.2) for $\mu=1$.

Our next result is an improvement of Theorem 1.3 which in turn gives a generalization as well as a refinement of Theorem 1.2.

THEOREM 1.5. If $p(z)=a_{n} z^{n}+\sum_{v=\mu}^{n} a_{n-v} z^{n-v}, 1 \leq \mu \leq n$, is a polynomial of degree $n$ having all its zeros in $|z| \leq k ; k \leq 1$ and $m=\min _{|z|=k}|p(z)|$, then for every real or complex number $\beta$ with $|\beta| \leq 1, r>0, p>1, q>1$ with $1 / p+1 / q=1$,

$$
\begin{aligned}
& n\left\{\int_{0}^{2 \pi}\left|p\left(e^{i \theta}\right)+\frac{\beta m e^{i(n-1) \theta}}{k^{n-\mu}}\right|^{r} d \theta\right\}^{1 / r} \\
& \quad \leq\left\{\int_{0}^{2 \pi}\left|1+k^{\mu} e^{i \theta}\right|^{p r} d \theta\right\}^{1 / p r}\left\{\int_{0}^{2 \pi}\left|p^{\prime}\left(e^{i \theta}\right)\right|^{q r} d \theta\right\}^{1 / q r} .
\end{aligned}
$$

REMARK 1.6. Letting $r \rightarrow \infty$ and $q \rightarrow \infty$ (so that $p \rightarrow 1$ ) in (1.6) and choosing the argument of $\beta$ suitably with $|\beta|=1$, it follows that, if $p(z)=a_{n} z^{n}+\sum_{v=\mu}^{n} a_{n-v} z^{n-v}$, $1 \leq \mu \leq n$, is a polynomial of degree $n$ having all its zeros in $|z| \leq k ; k \leq 1$, then

$$
\max _{|z|=1}\left|p^{\prime}(z)\right| \geq \frac{n}{1+k^{\mu}}\left\{\max _{|z|=1}|p(z)|+\frac{1}{k^{n-\mu}} \min _{|z|=1}|p(z)|\right\} .
$$

Inequality (1.7) was recently proved by Aziz and Shah [2].

2. Lemmas. For the proof of Theorem 1.5 we will make use of the following lemmas.

LEMmA 2.1 (see Aziz and Shah [2, Lemma 2]). If $p(z)=a_{n} z^{n}+\sum_{v=\mu}^{n} a_{n-v} z^{n-v}$ is a polynomial of degree $n$ having all its zeros in $|z| \leq k \leq 1$, then

$$
\left|q^{\prime}(z)\right| \leq k^{\mu}\left|p^{\prime}(z)\right| \quad \text { for }|z|=1,1 \leq \mu \leq n,
$$

where here and throughout $q(z)=z^{n} \overline{p(1 / \bar{z})}$. 
LEMMA 2.2 (see Rather [6]). If $p(z)=a_{n} z^{n}+\sum_{v=\mu}^{n} a_{n-v} z^{n-v}, 1 \leq \mu \leq n$, is a polynomial of degree $n$ having all its zeros in $|z| \leq k \leq 1$, and $m=\min _{|z|=k}|p(z)|$, then

$$
k^{\mu}\left|p^{\prime}(z)\right| \geq\left|q^{\prime}(z)\right|+\frac{m n}{k^{n-\mu}} \quad \text { for }|z|=1
$$

\section{Proof of theorems}

Proof OF TheOrem 1.3. Suppose that $p(z)$ has all its zeros in $|z| \leq k \leq 1$, therefore, by Lemma 2.1 we have

$$
k^{\mu}\left|p^{\prime}(z)\right| \geq\left|q^{\prime}(z)\right| \text { for }|z|=1 .
$$

Also $q(z)=z^{n} \overline{p(1 / \overline{\mathcal{z}})}$ so that $p(z)=z^{n} \overline{q(1 / \overline{\mathcal{z}})}$, we have

$$
p^{\prime}(z)=n z^{n-1} \overline{q\left(\frac{1}{\bar{z}}\right)}-z^{n-2} \overline{q^{\prime}\left(\frac{1}{\bar{z}}\right)} .
$$

Equivalently,

$$
z p^{\prime}(z)=n z^{n} \overline{q\left(\frac{1}{\bar{z}}\right)}-z^{n-1} \overline{q^{\prime}\left(\frac{1}{\bar{z}}\right)}
$$

which implies

$$
\left|p^{\prime}(z)\right|=\left|n q(z)-z q^{\prime}(z)\right| \quad \text { for }|z|=1
$$

Using (3.1) in (3.4) we get

$$
\left|q^{\prime}(z)\right| \leq k^{\mu}\left|n q(z)-z q^{\prime}(z)\right| \quad \text { for }|z|=1 ; 1 \leq \mu \leq n .
$$

Since $p(z)$ has all its zeros in $|z| \leq k \leq 1$, by the Gauss-Lucas theorem all the zeros of $p^{\prime}(z)$ also lie in $|z| \leq 1$. This implies that the polynomial

$$
z^{n-1} \overline{p^{\prime}\left(\frac{1}{\bar{z}}\right)}=n q(z)-z q^{\prime}(z)
$$

has all its zeros in $|z| \geq 1 / k \geq 1$.

Therefore, it follows from (3.5) that the function

$$
w(z)=\frac{z q^{\prime}(z)}{k^{\mu}\left(n q(z)-z q^{\prime}(z)\right)}
$$

is analytic for $|z| \leq 1$ and $|w(z)| \leq 1$ for $|z| \leq 1$. Furthermore $w(0)=0$. Thus the function $1+k^{\mu} w(z)$ is subordinate to the function $1+k^{\mu} z$ in $|z| \leq 1$. Hence by a well-known property of subordination [3] we have for $r>0$ and for $0 \leq \theta<2 \pi$,

$$
\int_{0}^{2 \pi}\left|1+k^{\mu} w\left(e^{i \theta}\right)\right|^{r} d \theta \leq \int_{0}^{2 \pi}\left|1+k^{\mu} e^{i \theta}\right|^{r} d \theta .
$$


Also from (3.7), we have

$$
1+k^{\mu} w(z)=\frac{n q(z)}{n q(z)-z q^{\prime}(z)}
$$

or

$$
|n q(z)|=\left|1+k^{\mu} w(z)\right|\left|n q(z)-z q^{\prime}(z)\right| .
$$

Using (3.4) and also $|p(z)|=|q(z)|$ in (3.10), we have

$$
n|p(z)|=\left|1+k^{\mu} w(z)\right|\left|p^{\prime}(z)\right| \text { for }|z|=1 .
$$

Combining (3.8) and (3.11) we get

$$
n^{r} \int_{0}^{2 \pi}\left|p\left(e^{i \theta}\right)\right|^{r} d \theta \leq \int_{0}^{2 \pi}\left|1+k^{\mu} e^{i \theta}\right|^{r}\left|p^{\prime}\left(e^{i \theta}\right)\right|^{r} d \theta \quad \text { for } r>0 .
$$

Now applying Hölder's inequality for $p>1, q>1$ with $1 / p+1 / q=1$ to (3.12), we get

$$
\begin{aligned}
& n^{r} \int_{0}^{2 \pi}\left|p\left(e^{i \theta}\right)\right|^{r} d \theta \\
& \quad \leq\left\{\int_{0}^{2 \pi}\left|1+k^{\mu} e^{i \theta}\right|^{p r} d \theta\right\}^{1 / p}\left\{\int_{0}^{2 \pi}\left|p^{\prime}\left(e^{i \theta}\right)\right|^{q r} d \theta\right\}^{1 / q} \text { for } r>0
\end{aligned}
$$

which is equivalent to

$$
\begin{aligned}
& n\left\{\int_{0}^{2 \pi}\left|p\left(e^{i \theta}\right)\right|^{r} d \theta\right\}^{1 / r} \\
& \quad \leq\left\{\int_{0}^{2 \pi}\left|1+k^{\mu} e^{i \theta}\right|^{p r} d \theta\right\}^{1 / p r}\left\{\int_{0}^{2 \pi}\left|p^{\prime}\left(e^{i \theta}\right)\right|^{q r} d \theta\right\}^{1 / q r} \quad \text { for } r>0
\end{aligned}
$$

which proves the desired result.

Proof of TheOrem 1.5. Since $p(z)$ has all its zeros in $|z| \leq k \leq 1$, therefore, by Lemma 2.2 we get

$$
k^{\mu}\left|p^{\prime}(z)\right| \geq\left|q^{\prime}(z)\right|+\frac{m n}{k^{n-\mu}} \quad \text { for }|z|=1,1 \leq \mu \leq n .
$$

Also by (3.4) for $|z|=1$, we have

$$
\left|p^{\prime}(z)\right|=\left|n q(z)-z q^{\prime}(z)\right| .
$$

Now using (3.15) for every complex $\beta$ with $|\beta| \leq 1$, we get

$$
\begin{aligned}
\left|q^{\prime}(z)+\bar{\beta} \frac{m n}{k^{n-\mu}}\right| & \leq\left|q^{\prime}(z)\right|+\frac{m n}{k^{n-\mu}} \\
& \leq k^{\mu}\left|p^{\prime}(z)\right| \\
& =k^{\mu}\left|n q(z)-z q^{\prime}(z)\right| \text { for }|z|=1 .
\end{aligned}
$$


Since $p(z)$ has all its zeros in $|z| \leq k \leq 1$, the result follows on the same lines as that of Theorem 1.3. Hence we omit the proof.

\section{REFERENCES}

[1] A. Aziz and N. Ahemad, Integral mean estimates for polynomials whose zeros are within a circle, Glas. Mat. Ser. III 31(51) (1996), no. 2, 229-237. MR 98d:30008. Zbl 874.30001.

[2] A. Aziz and W. M. Shah, An integral mean estimate for polynomials, Indian J. Pure Appl. Math. 28 (1997), no. 10, 1413-1419. MR 99a:30005. Zbl 895.30004.

[3] E. Hille, Analytic Function Theory. Vol. II, Introductions to Higher Mathematics, Ginn and Company, New York, 1962. MR 34\#1490. Zbl 102.29401.

[4] M. A. Malik, On the derivative of a polynomial, J. London Math. Soc. (2) 1 (1969), 57-60. MR 40\#2827. Zbl 179.37901.

[5] _ An integral mean estimate for polynomials, Proc. Amer. Math. Soc. 91 (1984), no. 2, 281-284. MR 85c:30007. Zbl 543.30002.

[6] N. A. Rather, Extremal properties and location of the zeros of polynomials, Ph.D. thesis, University of Kashmir, 1988.

[7] P. Turan, Über die Ableitung von Polynomen, Compositio Math. 7 (1939), $89-95$ (German). MR 1,37b.

K. K. Dewan: Department of Mathematics, Faculty of Natural Sciences, Jamia Millia ISLAMIA, NEW DELHI-110025, INDIA

E-mail address: kkdewan123@rediffmai1.com

AbDullah Mir and R. S. YADAV: DePaRtment of MAthematics, FACUlty of NATURAL SCIENCES, JAMIA Millia ISLAMia, NeW DELHI-110025, INDIA 


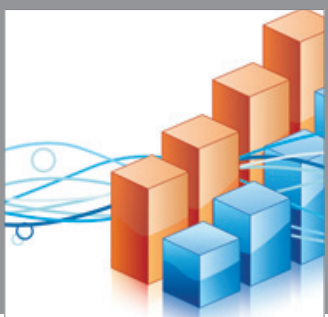

Advances in

Operations Research

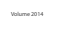

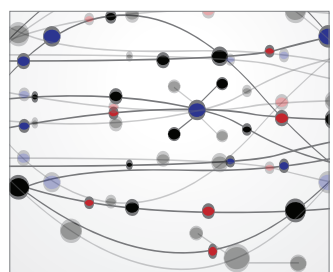

\section{The Scientific} World Journal
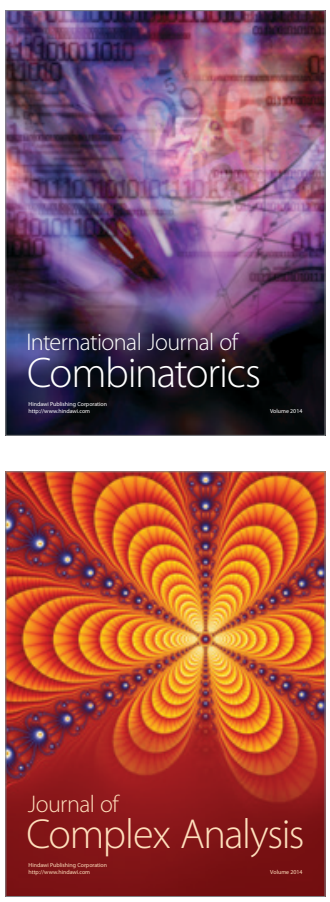

International Journal of

Mathematics and

Mathematical

Sciences
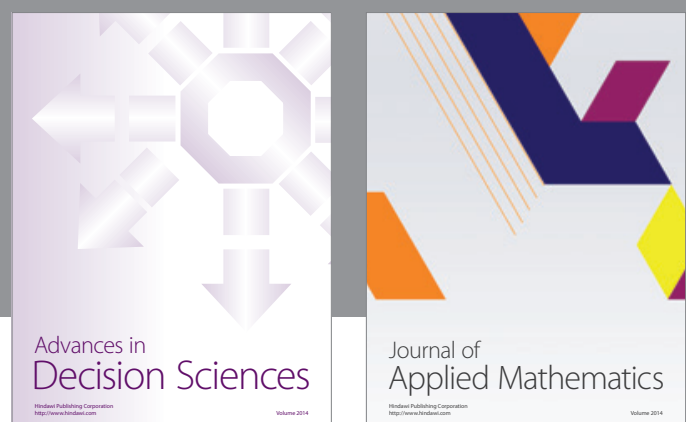

Journal of

Applied Mathematics
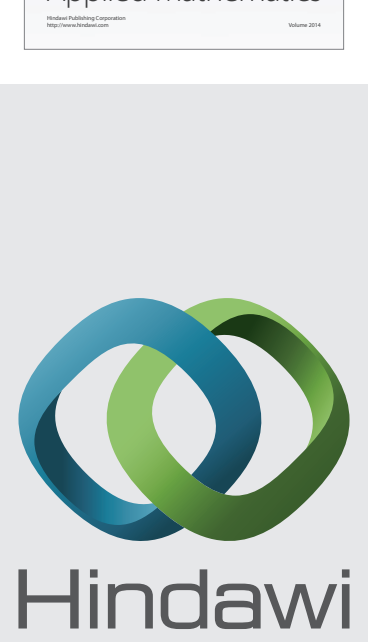

Submit your manuscripts at http://www.hindawi.com
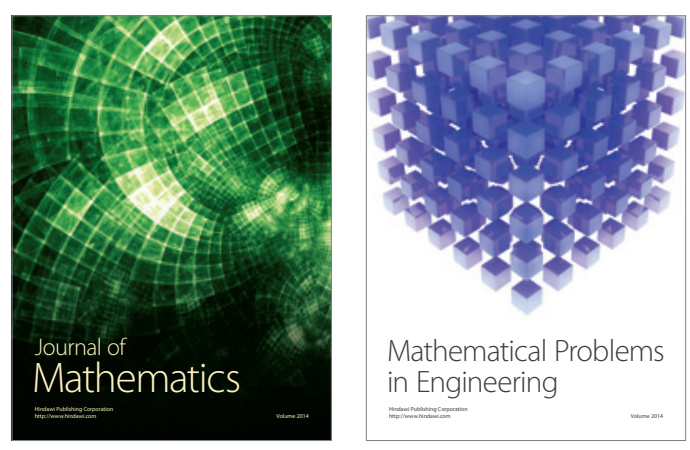

Mathematical Problems in Engineering
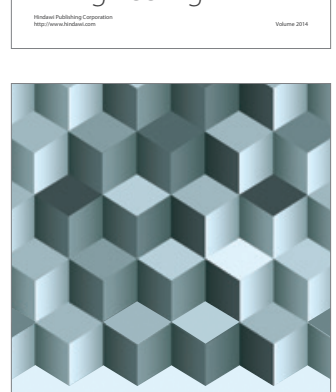

Journal of

Function Spaces
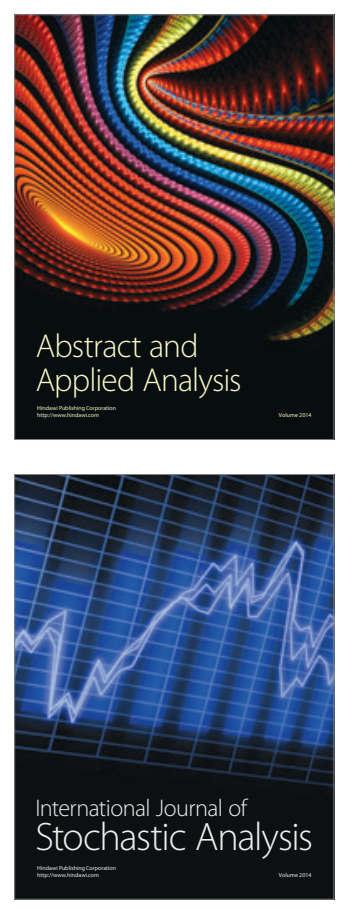

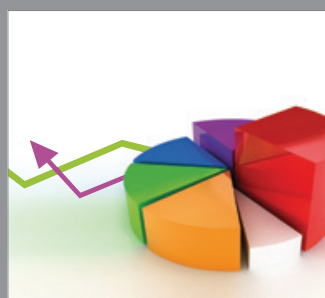

ournal of

Probability and Statistics

Promensencen
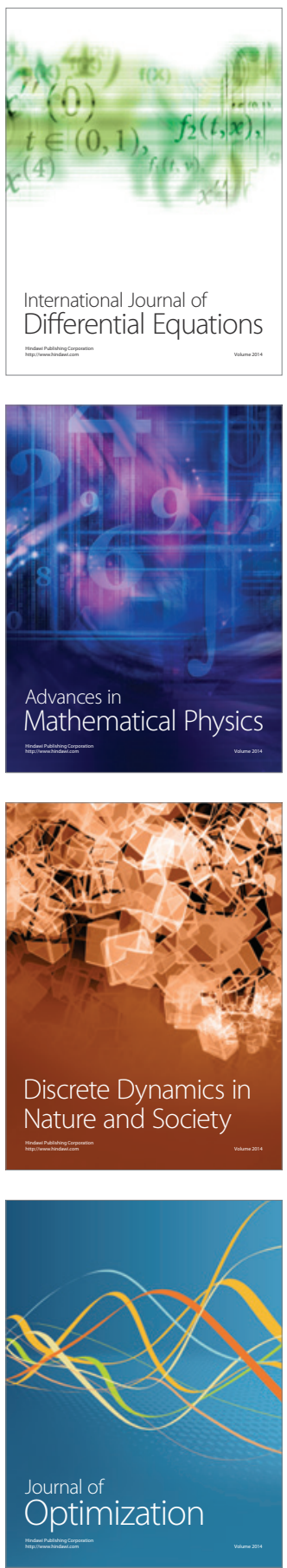